\title{
Las asociaciones profesionales de sociología en Argentina y las disputas por la "profesión"1
}

\author{
Cecilia Carrera $^{2}$
}

Fecha de recepción: 26 de agosto de 2019

Fecha de aprobación: 11 de octubre de 2019

Fecha de publicación: 31 de diciembre de 2019

\begin{abstract}
Resumen
Este escrito se refiere a las asociaciones profesionales de sociólogas y sociólogos en Argentina. El proceso de profesionalización y más concretamente la definición de qué es "dedicarse a la sociología" ha tenido un importante espacio en el desarrollo de la sociología local, generando debates y posiciones enfrentadas. Sin embargo, en los numerosos trabajos dedicados a la historia de la sociología y a la inserción profesional y laboral de sociólogxs, estas organizaciones reciben escasa atención. En este artículo describo algunas actividades que desarrollaron las organizaciones profesionales generadas desde la segunda mitad de la década de 1970. El propósito es explorar el lugar que estas organizaciones y sus participantes han tenido y tienen en el proceso de profesionalización de sociólogxs y en la construcción y disputa por los sentidos de la profesión en el país. Para ello, reconstruyo las perspectivas de algunxs de sus integrantes y sus contextos de producción. Procuro mostrar que, si bien quienes integraron e integran las asociaciones profesionales desde los años 70 no han participado activamente de los debates sobre la historia y génesis de la sociología que tienen protagonismo entre investigadores, sí han buscado
\end{abstract}

1 El presente artículo forma parte de la investigación doctoral (Doctorado en ciencias sociales de la Universidad de Buenos Aires, Argentina) de la autora, que se encuentra en proceso y cuyo título es "Profesión, ocupación y formas de contar la historia. Etnografía sobre el conflicto por la profesionalización de sociólogxs en Argentina". Deseo agradecer a lxs sociólogxs que me recibieron en la sede del Consejo de Profesionales de Sociología, Colegio de Sociólogos de la Provincia de Buenos Aires y Asociación de Sociólogos de la República Argentina, para dialogar sobre sus actividades e intereses, y darme acceso al material de archivo de esas instituciones. En particular, mi agradecimiento con Alejandro Terriles. También agradezco a Catalina Wainerman y Alicia Palermo por su tiempo y a Diego Pereyra y Pedro Blois por el diálogo siempre abierto.

2 Magíster en Ciencias Sociales, Facultad de Humanidades y Ciencias de la Educación de Universidad Nacional de La Plata, Argentina. Facultad de Humanidades y Ciencias de la Educación, Universidad Nacional de La Plata y Centro de Antropología Social, Instituto de Desarrollo Económico y Social La Plata, Argentina. Contacto: mcecilia.carrera@yahoo.com.ar 
instalar sus maneras de entender y desarrollar la profesión, disputando con otros actores.

Palabras clave: sociólogxs, profesión, asociaciones profesionales, sociología

\title{
Professional associations of sociologists in argentina and disputes over the "profession"
}

\begin{abstract}
This text refers to professional associations of sociologists in Argentina. The professionalization process and, more specifically, the definition of what it means "to work as a sociologist" has had an important space in the development of local sociology, producing debates and confronting positions. Nevertheless, these organizations have received limited attention in numerous publications dedicated to the history of sociology and the professional insertion of sociologists. This article describes some activities carried out within the professional organizations created since the mid-1970s. The objective is to explore the role these organizations and its participants have had in sociologist professionalization process and in the construction and dispute over the profession's purpose in the country. To this end, this article reconstructs some of the members' perspectives and their production contexts, trying to show that, while the people who have joined the professional associations since the 1970s have not actively participated in the debates on the history and genesis of sociology, prominent among researchers, they have set out to establish their ways of understanding and developing the "profession" through disputes with other key players.
\end{abstract}

Key words: sociologists, profession, professional associations, sociology

\section{As associações professionais de sociologia na argentina e as disputas pela "profissão"}

\begin{abstract}
Resumo
Este artigo se refere às associações profissionais de sociólogas e sociólogos na Argentina. O processo de profissionalização e, mais concretamente a definição do que é "dedicar-se à sociologia" ocupa um espaço importante no desenvolvimento da sociologia local, gerando debates e posições contraditórias. No entanto, nos inúmeros trabalhos dedicados à história da sociologia e à inserção profissional e laboral de sociólogxs, estas organizações recebem escassa atenção. Neste artigo descrevo algumas atividades desenvolvidas pelas organizações profissionais geradas desde a segunda metade da década de 1970. O objetivo é explorar o lugar que estas organizações e seus participantes ocuparam e ocupam no processo de profissionalização de sociólogxs e na construção e disputa pelos sentidos da profissão no país. Para isto, reconstruo as perspectivas de alguns de seus integrantes e seus contextos de produção. Procuro mostrar que, embora aqueles que integraram e integram as associações profissionais desde os anos 70 não participaram ativamente dos debates sobre a história e gênesis da sociologia que possuem protagonismo entre investigadores, sim
\end{abstract}


buscaram instalar suas formas de entender e desenvolver a profissão, disputando com outros agentes.

Palavras-chave: sociólogxs- profissão- associações profissionais- sociologia

\section{Introducción}

Este escrito se refiere a las asociaciones profesionales de sociólogas y sociólogos en Argentina. En los numerosos trabajos dedicados a la historia del campo sociológico local y a la inserción profesional y laboral de sociólogxs, estas organizaciones reciben escasa atención. Esta afirmación general requiere, sin embargo, establecer algunas excepciones. La Sociedad Argentina de Sociología (SAS) y la Asociación Sociológica Argentina (ASA), en su conformación, actividades y desarrollo, han sido analizadas con profundidad en algunas investigaciones sobre la historia de la sociología en Argentina y su inscripción en América Latina, un punto que retomaré más adelante (Blanco, 2006; Pereyra, 2005).

También se ha dado lugar a analizar las discusiones que algunos sociólogos en particular, de nombres resonados, tuvieron en relación a las inserciones profesionales o los "usos aplicados"3 de la sociología. Por ejemplo, Blois (2012) reconstruye algunas posiciones de quienes promovían la sociología como profesión, "una profesión como cualquier otra" (p.17) entre las décadas de

3 De aquí en adelante, las comillas se introducen para indicar expresiones propias de lxs actores, expresiones nativas; esto es, las formas que lxs actores emplean para hablar de sí mismxs, de su trabajo, y del trabajo de otrxs sociólogxs. Estas formas están marcadas localmente (Pennycook, 2006). Siguiendo a Pennycook (2010), entiendo al lenguaje como forma de organizar la vida social, de dar sentidos a las prácticas y relaciones que conforman esa vida social y que, al mismo tiempo, es organizado por esa vida social y local. Según el autor, "entender el lenguaje como práctica local implica que éste es parte de la actividad social y local y que ambos, localidad y lenguaje, emergen de las actividades en que están involucrados" (p. 128. La traducción es propia). Las prácticas de lenguaje de mis interlocutores en el campo (que incluyen el habla y la escritura en diversos contextos y situaciones) son locales porque están atravesadas por el espacio y el tiempo, por la historia de la sociología, de las instituciones que integran y de los grupos dentro de ellas (Pennycook, 2010). Las comillas, entonces, resultan un recurso para marcar la localidad, en términos de Pennycook, de ciertas formas de nombrar -incluso cuando las palabras empleadas son de uso corriente en otros contextos. 
1960 y 1970: Manuel Mora y Araujo, José Luis De Imaz, Torcuato Di Tella que se enfrentaban a quienes sostenían que la sociología debía ser una práctica solamente académica o solamente comprometida con el cambio político.

Blois también hace una breve reseña de la creación del Colegio de Graduados de Sociología (CGS) en Buenos Aires en 1975 y de algunas de sus actividades entre los años 70 y los 80 . El colegio fue, según Blois (2018), "fruto del esfuerzo de un conjunto de sociólogos preocupados por el desarrollo profesional de la disciplina" (p. 200), y durante la dictadura "se constituyó como un espacio de reunión de varios de los sociólogos que habían sido expulsados de la Carrera"(Blois, 2012, p.94). Pero, ni esta organización, ni las que se crearon después - algunas pocos años más tarde y en diferentes lugares del país-, ni tampoco lxs sociólogxs que participaron o participan en ellas suelen ser incorporados en los análisis sobre la profesionalización, como actores que sostienen perspectivas y posiciones específicas, despliegan acciones, disputan la cuestión de la profesión, la formación y el conocimiento de lxs sociólogxs.

Esto llama la atención porque, desde 1975, año en que se creó el CGS en Buenos Aires, estas instituciones desarrollan actividades relacionadas con lo que hacen lxs sociólogxs cuando se insertan profesionalmente en diversos espacios laborales. Por ejemplo, el dictado de cursos sobre temas que las carreras de grado no ofrecían u ofrecen y que tienen que ver con saberes relacionados a las actividades laborales (realización de encuestas, diseño de políticas, utilización de programas para análisis de datos), la promoción de leyes que regulan el ejercicio en distintas jurisdicciones y la creación de colegios que controlan el ejercicio.

En este artículo describo algunas actividades que desarrollaron las organizaciones profesionales generadas desde la segunda mitad de la década de la década de 1970. El propósito es explorar el lugar que las asociaciones y sus participantes han tenido y tienen en el proceso de profesionalización de sociólogxs, y en la 
construcción y disputa por los sentidos de "la profesión" en el país. Para ello, busco reconstruir las perspectivas de algunos de sus integrantes y los contextos en que esas perspectivas tienen lugar.

Esta indagación forma parte de una investigación etnográfica en la que me propongo explorar prácticas y sentidos asociados a la profesión de sociólogxs en Argentina. Los objetivos de esta etnografía son, por un lado, comprender la valorización del trabajo de quienes desarrollan actividad profesional por fuera de ámbitos académicos y, por otro, identificar las disputas relativas a las formas de clasificar, ordenar y categorizar la actividad laboral de lxs sociólogxs, comprendidxs por ellxs mismxs y por otrxs como "intelectuales", "académicos", "técnicos", "profesionales".

En un contexto en el que la mayoría de lxs sociólogxs trabaja en organismos del Estado y buena parte de ellxs circula entre lugares de trabajo (entre otros, organismos estatales, universidades públicas y privadas, empresas privadas, consultoras de mercado y opinión, ONGs) (Blois, 2012; Rubinich \& Beltrán, 2010), algunas asociaciones profesionales y algunas investigaciones han buscado mostrar que, en la carrera de Sociología de la Universidad de Buenos Aires (UBA) - primera carrera de sociología del país y que dio origen a la definición de un espacio de profesionalización de la sociología-, se omite el problema de la inserción laboral de lxs graduadxs e incluso se "censura" su inserción fuera de la academia (Blois, 2012; Grupo Taller Pensar la Facultad, 2009). Esta "censura" suele proceder desde perspectivas que asumen que la sociología debe ser una práctica "crítica" y "transformadora" que no esté "al servicio de los poderosos" y que, fuera de los ámbitos académicos, el trabajo de lxs sociólogxs queda definido por los objetivos del empleador. Así, tales posiciones reivindican una representación del sociólogo que construye conocimiento "crítico" sobre la sociedad en condiciones de autonomía-la que ofrecerían los espacios académicos- y producen formas de desvalorización del "trabajo profesional". ¿De qué maneras participan las asociaciones profesionales y quienes las integran de los procesos 
y disputas relacionadas con la valoración del trabajo y "la profesión"?

El trabajo de campo desarrollado se compone de una variedad de actividades, del análisis etnográfico de una multiplicidad de materiales; observación y participación en paneles organizados y/o dictados por sociólogas y sociólogos; seminarios internos de equipos de investigación sobre profesionalización; presentaciones de libros; visitas a asociaciones profesionales; entrevistas con sociólogas de primeras generaciones graduadas de Sociología, investigadores de la historia de la sociología y de la inserción profesional, miembros de asociaciones profesionales actuales y disueltas; sociólogxs que trabajan o trabajaron en áreas del Estado y en consultoras políticas; trabajo con documentos y publicaciones escritas y audiovisuales: textos de historia de la sociología en Argentina, ensayos e investigaciones de sociólogxs considerados relevantes en el desarrollo del conocimiento sociológico, de las cátedras y carreras de sociología y de los institutos de investigaciones de la disciplina; documentos institucionales de las carreras de sociología; actas y documentos de algunas asociaciones profesionales; entrevistas realizadas a sociólogas y sociólogos publicadas en libros, revistas y sitios web.

\section{Algunas orientaciones teóricas}

Numerosos autores (Freidson, 2001; González Leandri, 1999, 2006;Johnson, 1995; Sarfatti Larson, 1980, 1988; Wagner, Weiss, Witrock \& Wollman, 1999) proponen entender a los procesos de profesionalización como uno de los más importantes principios de organización social durante el siglo XX. Algunos de ellos definen a la profesión como una de las formas históricamente posibles de control institucionalizado de una ocupación, cuestionando los enfoques que la vinculan con la naturaleza inherente a cierto tipo de ocupaciones basadas en el uso de conocimientos complejos. Johnson (1995) afirma que el proceso histórico de institucionalización de las profesiones es inescindible del proceso de 
consolidación de los estados modernos y que, por ello, las profesiones son "elementos fundamentales en la constitución de los objetos de la política. Debido a su papel en la identificación de nuevos problemas sociales, en la construcción y dirección de los medios, instrumentos e instituciones para solucionarlos" (González Leandri, 1999, p. 66). ¿Qué sentidos fue adquiriendo entonces la profesión para lxs sociólogxs argentinxs, y de qué maneras han buscado y buscan jugar un papel en la identificación de nuevos problemas sociales y en la construcción de los medios para solucionarlos?

"Profesión", "profesional" y "ocupación" son, además, categorías nativas que adquieren sentidos localmente construidos que los diversos actores disputan. En este marco, cobra interés considerar el caso particular de lxs sociólogxs en Argentina donde, mientras algunxs se esfuerzan por persuadir al Estado y la sociedad de la necesidad de sus conocimientos y servicios, otrxs niegan la profesionalización que no sea académica como vía legítima de carrera sociológica (Beltrán, 2010; Blois, 2012, 2018;Tortti \& Chama, 2003).

El término profesión tiene una característica. Es un concepto que la sociología y la historia han desarrollado y debatido abundantemente, y, al mismo tiempo, es un objeto de disputa, una etiqueta por la que quienes realizan diversas ocupaciones luchan por ganar, ya que se identifica con el prestigio, el reconocimiento, los privilegios y el poder. Entonces, determinar si una ocupación es o no una profesión tiene que ver con luchas de legitimación de la ocupación (Freidson, 2001). La literatura sobre profesiones tiende, en general, a entender a la profesión como un estatus que la mayoría de las ocupaciones buscarían alcanzar. Por ello, es interesante que el otorgamiento de la condición de "profesión" para la sociología y de "profesionales" para lxs sociólogxs, lejos de significar claramente la lucha por el mejoramiento del prestigio y posición económica, ha sido un asunto de importantes disputas en las cuales las posiciones históricamente más legitimadas abogaban por rechazar el estatus de "profesional", y adoptar 
las características de "intelectuales" y/o "académicos" (Blois, 2012;Rubinich\&Beltrán, 2010).

Estas tensiones en torno a la valoración y desvalorización de la profesión y del trabajo de sociólogxs como profesionales atraviesan el derrotero de las asociaciones, colegios y federaciones en las que se centra este artículo. Estas instituciones no corresponden con la imagen trazada en los estudios que construyen una mirada, utilizando los términos propuestos por Julia Evetts, "optimista" y "positiva" hacia las profesiones y al profesionalismo como un "valor ocupacional" (Evetts, 2012). Algunas de las principales organizaciones profesionales que analizo aquí buscaron y buscan erigirse en las proveedoras de códigos éticos para el ejercicio profesional, así como construir un discurso sobre "la profesión" que logre convocar a lxs sociólogxs y construir poder de intervención en la mercado laboral. Veremos que encuentran persistentes dificultades para lograr esa posición. Por ello, el análisis del lugar de las asociaciones en el proceso de organización de la profesión de sociólogx en Argentina debe tomar en cuenta que estas han construido en distintos momentos un lugar de cierta importancia, que en otros momentos se vio muy disminuida. En cualquier caso, resulta claro que desde que se crearon las primeras asociaciones, lxs sociólogxs que las integran discuten en el terreno de una pregunta cuya formulación aún genera controversias en el campo sociológico argentino: ¿qué es dedicarse a la sociología?

\section{¿Qué es dedicarse a la sociología?}

El proceso de profesionalización de sociólogxs en Argentina ha estado atravesado, al menos desde los años 50, por debates, desacuerdos y definiciones sobre qué es dedicarse a la sociología. La pregunta y los intentos por responderla han producido conflictos y posicionamientos enfrentados. Uno de esos conflictos tuvo como protagonistas, en las décadas de 1950 y 1960, a Gino Germani, quien se presentaba y era reconocido como un "soció- 
logo moderno" y para algunxs "el primer sociólogo" argentino, y a Alfredo Poviña, abogado cordobés que encarnaba una figura del sociólogo que se había ido forjando desde inicios de siglo XX y cuya actividad principal era la enseñanza de la sociología en universidades y la escritura de textos destinados a ser insumos de enseñanza. Poviña lideraba un grupo de sociólogxs de perfil similar, la mayoría de lxs cuales se desempeñaban como docentes en universidades del interior del país.

Buena parte del conflicto entre ambos sociólogos, y quienes trabajaban con ellos, se desplegó en la creación y desarrollo de las dos primeras asociaciones de sociología del país, las SAS y la ASA. La SAS fue creada en 1959 por Poviña; y Gino Germani, por su parte, creó un año después la ASA como espacio desde el cual disputar a la SAS el control de redes internacionales y ámbitos de trabajo bajo la idea de que el "sociólogo profesional" es quien se dedica de tiempo completo a la investigación social empírica ${ }^{4}$.

Ambas asociaciones, y particularmente sus líderes, disputaban la representación de lxs sociólogxs del país y control de las redes nacionales e internacionales que involucraban asociaciones regionales e internacionales, así como organismos de investigación, desarrollo de conocimiento social y elaboración de políticas. Disputaban "el control de un mismo campo intelectual; [Poviña y Germani] pretendían para sí la identidad de sociólogos y ambos aspiraban a representar nacional e internacionalmente a la disciplina" (Blanco, 2006, p.219).

\footnotetext{
$4 \quad$ Vale aclarar que retomar la disputa entre estos sociólogos no tiene el objetivo de desconocer otras figuras de la sociología argentina, incluso anteriores a Germani y Poviña. Lo que busco es dar relevancia a esta disputa en particular porque se desarrolló en buena parte a través de la organización de asociaciones de sociólogxs, que hasta el momento no existían en Argentina.
} 
Estas asociaciones y la contienda ${ }^{5}$ entre ellas fueron importantes en el plano de la profesionalización, porque representaban dos visiones sobre qué es dedicarse a la sociología. Eliseo Verón, autor de una de las primeras historias de la sociología argentina y, además, docente en la carrera de Sociología de la UBA desde sus primeras etapas, recuperó en 1974 el impacto de esta disputa entre asociaciones y sus líderes:

No hay que olvidar que [en la década del 60] persistía en el país un importante grupo de sociólogos representantes de orientaciones que se podrían llamar "precientificistas". Este grupo controlaba la Sociedad Argentina de Sociología, con sede en Córdoba, presidida por Alfredo Poviña. Aunque algunos de los sociólogos "modernos" pertenecen inicialmente a ella, se aglutinan después en la Asociación Sociológica Argentina, fundada en 1960 bajo la presidencia de Gino Germani, produciéndose así, en el plano de la profesionalización, una polarización clara entre los "tradicionales" y los "modernos". Una de las acusaciones que estos últimos dirigían a la Sociedad Argentina de Sociología era que reconocía como sociólogos a quienes no lo eran. (...) La Asociación de los "modernos" era muy clara en sus estatutos: la ASA tiene por fin " (...) proteger en el país la acción científica en la actuación profesional de los sociólogos (...) y tiene por socios activos a aquellos que posean título universitario específico" o bien "que posean título universitario en otra especialidad, cuando hayan producido contribuciones de nivel científico en Sociología y además tengan dedicación exclusiva". (Verón, 1974, p.41)

Esta disputa no se dio solamente en Argentina, sino que tuvo despliegue internacional, expresado en la oposición entre la International Sociological Association y el Institut Internacional de Sociologie (Blanco, 2006; Pereyra, 2005). 
¿Quién/es, desde qué posiciones, reconocen a otrxs como sociólogxs y son reconocidxs como tales? Verón ubicaba lo que llamó "polarización" entre "modernos" y "tradicionales" en el plano de la profesionalización, que parece aquí ligarse a una lucha por definir qué es dedicarse a la sociología.

Desde ASA, Germani y sus colaboradores apelaron a las nociones de "profesión" y "profesional" para generar una diferencia y una oposición entre "sociólogos" y personas que enseñan sociología pero que no se dedican a ella. A partir de allí, la relación de la sociología y lxs sociólogxs con las ideas, prácticas e identificaciones asociadas a "profesión" y "profesionalización", será materia de disputas entre diversos actores que se presentan como sociólogxs. Y la definición de qué significa dedicarse a la sociología, cuáles son los conocimientos y actividades que caracterizan lo que hacen, será materia de desacuerdos y una manera de definir posiciones, diferenciaciones y categorizaciones hasta el presente.

El modo de desplegar esas luchas que se ha consolidado en el campo sociológico local tiene como eje las diferentes interpretaciones de la historia de la sociología. Estas interpretaciones proponen clasificaciones y ordenamientos del campo de la sociología, principalmente a través de los relatos y explicaciones que se construyen sobre su génesis. Se trata de la construcción de genealogías enfrentadas, más o menos, abiertamente, que marcan itinerarios, nombres, obras, momentos que componen una interpretación de cómo se constituye el campo de la sociología y el problema de la profesionalización.

De manera muy breve, puede decirse que la genealogía más extendida es desarrollada por sociólogxs que ubican la fundación de la sociología y el surgimiento de la profesión en la creación de la carrera de la UBA en 1957 y en la figura de Gino Germani como "pionero". La idea de "creación" es central en los textos y relatos que sostienen esta perspectiva (Izaguirre, 2010; Jorrat \& Sautu, 1992, Mera \& Rebón, 2010), porque no se refiere simple- 
mente a la institucionalización de una carrera sino a la formación y "entrenamiento" de sociólogxs como "investigadores empíri$\cos ^{\prime \prime}$ y "expertos en problemas sociales"; a la "delimitación de la sociología como campo disciplinario", es decir a la "fundación" de una "nueva" sociología, que buscó erigirse en la "verdadera" sociologíaPero quienes, siendo estudiantes y docentes jóvenes de la misma carrera de Sociología de la UBA de la cual Germani era director, estuvieron en los años 60 al frente de una serie de cuestionamientos a la orientación que tenía la enseñanza, oponen otra genealogía. Algunxs sociólogxs se identifican en el presente con un "nosotros" al que dan continuidad desde las primeras protestas como estudiantes y luego ubican en la conformación de las llamadas Cátedras Nacionales desde el año 1967. La construcción de ese "nosotros" tiene su contracara en la identificación de un "ellos", que una socióloga define como "los hijos predilectos" de Germani (Entrevista a Alcira Argumedo. Maderna, 2000, p. 483). Quienes se identifican con esta genealogía reconocen como primerxs sociólogxs a ensayistas y pensadores sociales anteriores a la creación de la carrera y a la instalación de la "sociología moderna" (Carrera, 2018). Entre ellos, a Esteban Echeverría, José Ingenieros y Ernesto Quesada.

Ambas genealogías se construyen y actualizan, dando lugar a posiciones enfrentadas sobre la sociología y la actividad de lxs sociólogxs, expresadas en acusaciones mutuas: unxs serían, por ejemplo, "cientificistas" e "imperialistas", mientras que lxs otrxs serían "sólo ensayistas" y han tendido a diluir la sociología en la política.

¿Participan lxs sociólogxs que integran las asociaciones profesionales en las discusiones genealógicas sobre la profesión y la sociología? ¿Qué perspectivas produjeron y producen respecto de qué es dedicarse a la sociología? Las descripciones y análisis que presento a continuación buscan avanzar en la comprensión de las maneras que estaxs sociólogxs participan del campo sociológico construyendo sentidos sobre la profesión. 


\section{Asociarse para "defender la sociología" y "fortalecer la organización gremial"}

La ASA se disolvió pocos años después de su creación y la SAS continuó funcionando hasta entrados los años 80. Ambas instituciones inaugurarán una forma de asociarse y disputar que volverá a darse en el campo sociológico argentino: la coexistencia de asociaciones que pretenden ejercer representación y control de un mismo campo y una misma comunidad.

Algunxs graduadxs de las carreras de sociología que habían comenzado a funcionar a mediados de la década de1950 (en la UBA y en otras universidades públicas y privadas) crearon luego otras asociaciones. Estas incorporaron una preocupación novedosa, que no radicaría en determinar quién puede o no ser considerado sociólogx, sino en cuál sería la "ocupación" de lxs sociólogxs y, más puntualmente, qué inserciones laborales podrían lograr.

El Colegio de Graduados de Sociología se creó en 1975 en Buenos Aires. En ese momento, lxs graduadxs de sociología provenían de la carrera de la UBA, de la Universidad Católica Argentina, Universidad de El Salvador, Universidad de Belgrano y Universidad Kennedy.

En 1974, el gobierno de Isabel Perón había intervenido las universidades públicas, separando de sus cargos a muchxs de lxs docentes de la carrera de Sociología, y en los años siguientes persiguió y mató a muchxs de ellxs. Durante la dictadura militar que le siguió, algunxs sociólogxs se exiliaron, otrxs fueron desaparecidxs y otrxs encontraron lugares de trabajo donde pudieron desarrollar investigación y enseñanza de la sociología: en carreras de sociología de universidades privadas (USal, UB, UCA), en institutos de investigación como el CIS-Di Tella, el Ides o el CEIL; en organismos del Estado; y en empresas privadas y agencias de consultoría y sondeos de opinión (Rubinich, 2010; Sidicaro, 1993). Todos estos espacios se mantuvieron e incluso crecieron en 
esos años y luego de finalizada la dictadura. Las consultoras de opinión y asesoría política se fueron constituyendo en espacios laborales importantes en las décadas posteriores, donde algunxs sociólogxs construyeron legitimidad para intervenir en la esfera pública y mediática como profesionales de la sociología (Vommaro, 2008).

Podemos leer la creación y el funcionamiento del CGS durante la segunda mitad de los años 70 en un escenario con dos características significativas: muchxs sociólogxs empezaban a incorporarse a espacios de ejercicio que no eran la universidad pública y, al mismo tiempo, había cada vez más graduadxs-la década del 60 fue una de las que registró mayor graduación- $y$, por eso, cada vez más sociólogxs buscando trabajo.

Alicia Palermo, una socióloga que participó del CGS desde los primeros años ${ }^{6}$, identifica su creación como una forma de "defender la sociología" en un contexto restrictivo:

Vos en ese momento no tenías ni participación en la universidad ni en ningún otro lugar porque no podías estar en ningún lado, lo único que estaba era el Di Tella. Y entonces [en el CGS] se empezó a reunir la gente que tenía intereses académicos o políticos también, pero planteaba una defensa de la sociología. Había que visibilizar al sociólogo y ponerlo en el ámbito profesional porque prácticamente no existía. (Entrevista, julio de 2019)

Quienes impulsaron la creación del colegio buscaban que sea un espacio común en un momento en el que un buen número de ellxs perdían sus puestos en las universidades y, también, un espacio que dé "visibilidad profesional al sociólogo" y favorezca la "jerarquización de la profesión", según puede leerse en documentos y declaraciones que analizaré en próximos apartados.

Alicia Palermo participó del CGS hasta su disolución en 1994; también en el Consejo de Profesionales de Sociología, hasta inicios de la década de 2010, y es socia fundadora de la Asociación Argentina de Sociología, desde 2009 hasta la actualidad. 
Así, si bien el CGS no fue, como vimos en el apartado anterior, la primera asociación profesional de sociología en Argentina, sí parece haber sido la primera que explícitamente buscaba ocuparse de los asuntos de inserción laboral de profesionales, partiendo de que la sociología y lxs sociólogxs no gozaban de alta visibilidad y jerarquización.

El colegio se financiaba con la cuota que pagaban quienes voluntariamente se asociaban y funcionó hasta 1994, año en el que se disolvió debido a "la grave situación económica financiera", según se expresa en las actas ${ }^{7}$, por la cual atravesaba.

Desde 1977, quienes participaban del CGS organizaron de forma anual las Jornadas de Sociología y, también, desde los primeros años, ofrecían cursos de "actualización profesional". La mayoría eran, según Blois (2018, p. 200) "de perfil técnico", dedicados a metodología de investigación y temas "aplicados" como salud, educación, trabajo. En las actas de la Comisión Directiva $(\mathrm{CD})^{8}$ del colegio que he relevado 9 , ya en los primeros años 90, los cursos que se ofrecían y tenían más inscriptos y/o que se proyectaban eran sobre temáticas como: Computación en Ciencias Sociales (a cargo de Ponciano Torales); Cómo se inicia una investigación (Néstor Cohen); Marketing profesional; Cómo se realiza una encuesta preelectoral (Julio Aurelio); Metodología y epistemología de la investigación científica (Juan Samaja ${ }^{10}$ ). Estos cursos se hacían todos los años y se dictaba más de uno por año. Los cursos fueron una de las actividades más importantes,

Acta N³93 del 27/6/1994.

8 Formaron parte de la Comisión Directiva del CGS entre 1991y 1994, entre otrxs: Ponciano Torales, Juan Villareal, Laura Asta, Liliana Torres, Alicia Palermo, Roberto Torkelsen, Norberto Zeller.

9 Tuve acceso a Actas del Colegio de 1983, abocadas a la constitución de la Federación Argentina de Sociólogos (que nucleaba a las asociaciones y colegios del país): 5 actas entre 1983 y 1985. También a las últimas actas del Colegio antes de su disolución, entre noviembre de 1991 y julio de 1994.

10 Este último se dictó en 1987, y consta en el currículum vitae de Jorge Elbaum, quien realizó el seminario como cursante. Disponible en: https://economicas. unlam.edu.ar/descargas/5_B003.pdf 
constantes y exitosas del CGS, lo cual puede evidenciarse en las evaluaciones positivas que se encuentran en las Actas de la CD de los cursos que se iban dictando.

También organizaban jornadas sobre temas que interpretaban de interés para sociólogxs en ejercicio. En 1991, hicieron jornadas denominadas "Estratificación social y estructura de clases" y en 1992 sobre "Políticas sociales". Se trataba de jornadas donde lxs miembros del colegio que asumían la organización armaban el programa de actividades, convocando a "expertos" a "reuniones preparatorias" y tenían mesas redondas y talleres. En las actas se mencionan algunxs de esxs expertxs: Beba Balvé, Ernesto Villanueva, Luis Rigal, Daniel Filmus, Aldo Isuani y Emilio Tenti.

Se hacían en la sede del CGS charlas a cargo de sociólogxs reconocidos nacional e internacionalmente, y algunxs extranjerxs. En las actas de CD del año 1992, por ejemplo, puede verse que llegaban propuestas para que algunas charlas se hicieran en la sede del colegio: "se ha ofrecido al colegio la posibilidad de realizar en nuestra institución una conferencia sobre marginalidad a cargo del sociólogo Robert Castell" (Acta 367, 17/11/1991).También dieron conferencias Daniel Bel, James Petras, Dora Barrancos, Atilio Borón y Catalina Wainerman.

A juzgar por esta intensa actividad, la institución había logrado visibilidad y reconocimiento por parte de la comunidad de sociólogxs. En los primeros años 90, que fueron sus últimos años de existencia, parece haber constituido un espacio en el que muchxs sociólogxs buscaban participar asociándose, ofreciendo cursos, conferencias, organizando jornadas o asistiendo a esos eventos. Se registra en la mayoría de las actas que cada mes se sumaban personas que solicitaban ser asociadas.

Hacia finales de la década del 70 e inicios de la siguiente, aún en dictadura, se crearon otras organizaciones de sociología en otros lugares del país: la Asociación de Graduados en Sociología de La Plata y Gran Buenos Aires, la Federación Provincial de Sociología, el Colegio de Graduados de Sociología de Santiago 
del Estero y el Colegio de Graduados de Sociología de San Juan. De manera que la actividad intensa y el lugar de cierto reconocimiento que había logrado el colegio se inició en la segunda mitad de la década del 70, pero se consolidó durante los años 80 y no involucró solo al CGS sino a otras organizaciones de graduadxs de sociología.

En una reseña institucional publicada en 2015, en la página web del Colegio de Sociólogos de la Provincia de Buenos Aires $(\mathrm{CSPBA})^{11}$, su autor y entonces presidente, Alejandro Terriles, se refiere al escenario de creación de estas instituciones:

Como parte de un amplio movimiento de resistencia a los múltiples embates que durante el denominado Proceso de Reorganización Nacional (1976-1983) obstaculizaron el desarrollo de la Sociología en nuestro país, surgieron un conjunto de Asociaciones de Graduados donde se nuclearon los profesionales universitarios que pretendían defender la carrera de grado y el ejercicio profesional contra los intentos desintegradores del campo por parte del totalitarismo de la dictadura militar.

Terriles entiende la creación de estas organizaciones, integradas "en un amplio movimiento", como formas de resistencia y defensa de la sociología y el ejercicio profesional en el contexto de la dictadura militar. En la misma reseña, se refiere a la vida de las organizaciones de la Provincia de Buenos Aires durante la década de los 80: "aquellos años fueron testigos de una prolífica actividad interna traducida en el crecimiento de la matrícula y en

11 El CSPBA fue creado por ley provincial en 1985. Sus antecedentes fueron la Asociación de Graduados en Sociología de La Plata y Gran Buenos Aires y la Federación Provincial de Sociología. El mismo año comenzó a funcionar en la UNLP una carrera de sociología destinada a estudiantes avanzados y graduados de otras carreras, pero ya existía la carrera de sociología de la UCALP y había sociólogxs graduados de la UBA y universidades privadas diversas que trabajaban en la Provincia. 
la generación de múltiples iniciativas tendientes a lograr visibilidad y reconocimiento por parte de toda la sociedad provincial".

En Córdoba, otro de los centros significativos de formación de sociólogxs durante el siglo XX, también se creó en 1984 una organización profesional que se sumaba a las ya mencionadas. Ada Caracciolo, una socióloga que participaba en actividades del Colegio de Buenos Aires, pero sobre todo más activamente en la organización del Colegio de Graduados y Especialistas en Sociología de Córdoba ${ }^{12}$, definió los primeros años de la vuelta de la democracia como años de "efervescencia reorganizativa" de los "revitalizados colegios profesionales" (Caracciolo, 2008).

La búsqueda de "defender la sociología" tenía sentido no sólo en Buenos Aires. Pero, ¿qué significaba "defender la sociología" desde las perspectivas de quienes participaban en estas organizaciones y cómo buscaban defenderla?

Una estrategia concreta se consolidó en noviembre de 1983, cuando los colegios y asociaciones existentes organizaron el I Congreso Nacional de Sociología en Buenos Aires. Durante el congreso, representantes de esas organizaciones acordaron crear una Federación Nacional de Sociólogos (FNS). El Colegio de Graduados de Sociología de San Juan, la Asociación de Graduados

12 El Colegio de Graduados y Especialistas en Sociología de Córdoba se creó en 1984, con el objetivo de, entre otros, promover la creación de la carrera de grado en la Universidad Nacional de Córdoba (UNC)-lo cual sucedió recién en 2017. El caso es particular porque es el único en el cual la institución profesional existe antes que la carrera de grado en la provincia. Esta inexistencia de carrera de grado no suponía ausencia de sociólogxs y menos de actividad relacionada a la sociología. En sus investigaciones sobre la trayectoria de Alfredo Poviña, Diego Díaz se refiere a la existencia de un espacio de "la sociología cordobesa", con sus actores, instituciones, dinámicas relacionales y luchas internas específicas, que podrían explicar en parte el hecho de que no se haya creado hasta hace pocos años una carrera de Sociología en uno de los más importantes centros universitarios del país, del que surgieron algunas de las figuras más activas del campo sociológico argentino, como Raúl Orgaz, el mismo Poviña y Juan Carlos Agulla. El Colegio tuvo actividad sistemática hasta la década del '90 (Caracciolo, 2008). Se llamó Colegio de Graduados y Especialistas en Sociología, porque lo que se había creado en la UNC era una titulación de especialización en sociología para graduadxs de otras carreras (Díaz, 2013). 
de Sociología de La Plata, el Colegio de Graduados de Sociología de Santiago del Estero, las comisiones promotoras del Colegio de Córdoba y Salta, "y con las adhesiones de organizaciones de Mar del Plata, Neuquén y otros puntos del país" (Acta $\mathrm{N}^{\circ} 1$ de la FNS), estuvieron allí para constituir esta Federación. El acta constitutiva expresa

la necesidad de fortalecer la organización gremial de todos los graduados en sociología y expresado la voluntad de: 1) constituirse en una Federación Nacional de Sociólogos formada por las entidades de graduados que asuma como objetivos básicos la defensa y jerarquización de la profesión, el fortalecimiento de los nucleamientos de base y su representación ante organismos públicos y privados nacionales e internacionales y la realización de todas las actividades que conduzcan a lograr un aporte más pleno y eficaz de la sociología como ciencia autónoma dentro de las ciencias sociales. 2) organizar anualmente un Congreso Nacional de Sociología. 3) Agotar las instancias que tiendan a la promulgación de los instrumentos normativos que regulan el ejercicio profesional. Asimismo, expresan la convicción de que estas acciones permitirán asegurar y defender el ejercicio profesional y contribuirán al conocimiento y estudio de los problemas de la comunidad argentina contribuyendo así en su solución. (Acta $\mathrm{N}^{\circ} 1$ de la FNS, 1983)

Así, la "defensa de la sociología" era la defensa de "la profesión" a través de, entre otras estrategias, la organización gremial y la generación de instrumentos de regulación del ejercicio. El acta también hace referencia a acuerdos previos que se habían alcanzado en una reunión en mayo de ese año en Santiago del Estero. A esa reunión se refiere Caracciolo (2008) en un escrito sobre el Colegio de Córdoba:

Esta reunión es decisiva. Por un lado instala en el debate cuestiones básicas: la reorganización institucional de la 
sociología, se impulsa predominantemente desde los colegios profesionales de Sociología, en anticipación a posibles acciones desde el interior de la universidad; la centralidad de la investigación en la consideración de la formación del sociólogo y la profesionalización de la sociología. (p.13)

Es significativo lo que recuerda Caracciolo, en cuanto pone en el centro de "la reorganización institucional de la sociología" a los colegios profesionales. Así, además de espacios reconocidos y visibilizados para que, por ejemplo, sociólogxs extranjeros que venían a Argentina dieran conferencias, los colegios profesionales parecen haber asumido (o buscado asumir) en los 80 un rol de "recomponer institucionalmente" a la sociología, después de los años de dictadura. Esta "recomposición institucional" tuvo como su primer objetivo, según podemos saber a través del acta de la FNS, la organización gremial y la "defensa y jerarquización de la profesión". Esto se relaciona con la novedad que introdujeron estas asociaciones creadas a partir de los 70: la preocupación por el trabajo de lxs sociólogxs y su organización como "profesionales" y, específicamente, como trabajadores, que requieren de un gremio que regule su actividad y la defienda en un mercado laboral.

\section{"Crear una ocupación". Legislación y asociación}

En 1982, tres sociólogas y dos sociólogos del CGS publicaron el informe de una investigación que habían realizado para conocer en qué estaban trabajando lxs sociólogxs en ese momento. El informe se tituló Espectro ocupacional del licenciado en sociología en el medio profesional argentino y fue el primer estudio sobre el asunto, todavía hoy citado y recuperado por investigadores del tema. Lxs autorxs fueron Ernesto Bialakowsky, Hilda de Martín, Raquel Macri, Daniel Filmus y Ada Caracciolo (1982) quienes, en la introducción, plantean que "han visualizado como una necesidad" analizar "la ocupación" que en esa misma introducción definen como "el dominio de un arte o una técnica para la ejecución 
de un trabajo determinado fruto de la división del trabajo social" (p. 2) y, también, como "el encadenamiento" entre el "oficio", "su nexo colectivo" y "la preparación formal" (p. 5).

Muestran también dos preocupaciones: una de ellas, vinculada al "valor de la profesión y su ejercicio para los profesionales". Afirman que

esta investigación, aunque de carácter empírico-descriptiva, se encuadra o enmarca en un proceso más amplio de autoreflexión. Autoconciencia necesaria, para quienes como nosotros el desempeño profesional constituye -a pesar de los obstáculos y del acontecimiento- una forma elemental de proveer a nuestro sustento, valorizarnos subjetivamente a través del mismo y servir a la comunidad en la medida de nuestras posibilidades. (Bialakowsky et al., 1982, p. 1).

Lxs autorxs, autoidentificadxs como "profesionales", sostienen que la profesión adquiere un lugar de centralidad al proporcionar satisfacciones no necesariamente ligadas a las recompensas materiales sino más bien a la vocación, la implicación y el compromiso con las tareas.

Considerando la importancia que adquiere la profesión para lxs profesionales, concluyen que la falta de identificación, la "desubicación" o abandono del rol profesional trae consecuencias individuales en la personalidad y sociales de anomia y alienación que ameritaban en ese momento un análisis de la situación de la sociología como profesión y de "los sociólogos y su espectro ocupacional" (Bialakowsky et al., 1982, p. 1). Al parecer, el reconocimiento que las asociaciones habían logrado al interior de la comunidad de sociólogxs y graduadxs de otras ciencias sociales no tenía correlato en un reconocimiento amplio "del sociólogo" que permitiera construir un "espectro ocupacional" satisfactorio.

Con esta cuestión está relacionada la segunda preocupación manifestada en el informe. Esta tiene que ver con lo que mencio- 
nan como las "características estructurales" que se han establecido en la sociología de las profesiones para definir una profesión: “1) creación de una ocupación de tiempo completo; 2) establecimiento de una escuela preparatoria; 3) formación de asociaciones profesionales y 4) formación de un código ético" (Bialakowsky et al., 1982, p. 2). Así evaluaban la situación de la sociología argentina:

Una rápida revista (...) nos permite comprobar que en tanto la carrera existe, (...) e igualmente existen asociaciones profesionales -p.e., el Colegio de Graduados en Sociología-, no es posible verificar un código ético explicitado y mínimamente consensual (instituido en alguna forma específica) destinado a la especialidad, igual dificultad encontramos, al menos a priori, de constatar la 'creación de una ocupación de tiempo completo'.

Dado que el establecimiento de un código de comportamiento partirá, o será dependiente, de los otros tres elementos, se hace inexcusable reflexionar sobre el último de los nombrados: la creación de una ocupación. (p. 2)

Lo que preocupaba a estxs sociólogxs es que buena parte de sus colegas no se dedicaba a tiempo completo a la sociología, pues el "espectro ocupacional" disponible no permitía que el sustento necesario fuera satisfecho exclusivamente por el trabajo profesional. Nos encontramos nuevamente con el interrogante de qué implica dedicarse a la sociología. Pero, en este caso, la dedicación "a tiempo completo" no es, como se establece en el estatuto de ASA, un requisito a partir del cual establecer quién es o no considerado sociólogo, sino un problema a resolver. Tal problema no es abordado en este informe recurriendo a tradiciones, genealogías y prácticas que ubiquen en una u otra concepción de la sociología a quienes se definen como sociólogxs, sino a la pregunta por la "ocupación". La dificultad para constituirla que evidencia el estudio era un asunto que, para estxs sociólogxs, importaba a las asociaciones profesionales como parte de la "defensa y jerarquización de la profesión". 
Pero desde el punto de vista de otrxs sociólogxs, la no dedicación a tiempo completo ponía en duda directamente su relación con el ejercicio de la sociología. Catalina Wainerman, una de las primeras egresadas de la carrera de Sociología de la UBA, conocida discípula de Gino Germani, referente de la sociología "moderna" y "empírica" que impulsó el sociólogo italiano, fue investigadora del Centro de Estudios de Población (CENEP) desde su creación en 1974 (Blois, 2019). Este Centro compartió sede edilicia con el CGS durante varios años, por lo que quienes formaban parte del colegio y lxs investigadorxs del CENEP compartían espacios cotidianamente, se encontraban allí, se conocían. Incluso, Wainerman dictó conferencias en el CGS y fue parte del Comité de Ética del Consejo de Profesionales de Sociología creado posteriormente. Durante una entrevista con esta socióloga, le pregunté cuál había sido su experiencia y sus vínculos con ambas instituciones. Wainerman recordó los años en que el CENEP y el CGS funcionaban en un mismo piso en un edificio de Buenos Aires y mencionó con cariño a algunas de las personas que participaban. También dijo que, aunque las veía frecuentemente, "nunca supe a qué se dedicaban esas personas. Pero seguro que a la sociología, no" (Entrevista, 5 de septiembre de 2018).

El recuerdo y valoración de Wainerman son significativos si los comprendemos como parte de una trama de posicionamientos construidos históricamente entre sociólogxs y grupos de sociólogxs, a partir de la pregunta por qué es dedicarse a la sociología. Las ideas y prácticas asociadas a qué es un "sociólogo profesional" y cuáles son las actividades y características que permiten que las personas sean así reconocidas continúan, en el presente, siendo objetos de posicionamientos diferenciales, reconocimientos y desconocimientos. La presencia y protagonismo que las asociaciones profesionales buscaron sostener desde mediados de los 70 y durante los 80 - y parecen haber conseguido- no es suficiente para que todxs quienes se identifican como sociólogxs reconozcan a quienes participaban en las asociaciones como parte de su misma profesión. 
Otra de las preocupaciones que se muestran en el informe de 1982, y que también se evidencian en los pasajes citados del acta constitutiva de la FNSde 1983, tiene que ver con el establecimiento de normas y estatutos de regulación del ejercicio profesional. Las asociaciones buscaron resolver ese aspecto por la vía de la legislación y la década del 80 fue prolífica en este aspecto. En 1985 se aprobaron las incumbencias profesionales para todas las carreras de sociología del país; también se sancionó la Ley 10.307 sobre el ejercicio profesional de la sociología de la Provincia de Buenos Aires, que creó el Colegio de Sociólogos de esa provincia.

Desde 1986, un grupo de integrantes del CGS comenzó a trabajar para que se promulgara una ley que regulara el ejercicio profesional de lxs sociólogxs, la cual fue sancionada en abril de 1988. Como parte de la Ley 23.553 de Ejercicio Profesional de la Sociología, se creó el Consejo de Profesionales de Sociología (CPS). Este Consejo, a diferencia del Colegio de Graduados, tiene atribuciones de control del ejercicio, matriculación de sociólogxs en actividad $-\mathrm{y}$ cobro de matrícula- e incorpora un Tribunal de Ética y Disciplina. Según esa ley, para desempeñarse como profesionales "en la Capital Federal, el Territorio Nacional de Tierra del Fuego, Antártida e Islas del Atlántico Sur"(Ley 23553, artículo $\left.1^{\circ}\right)^{13}$, lxs sociólogxs tienen la obligación de matricularse en el Consejo, y los empleadores la obligación de solicitarle la matrícula para emplearlos.

El Consejo empezó a funcionar en 1990, año en que se eligió a su Comisión Directiva ${ }^{14}$. El CGS y el CPS funcionaron si-

El alcance jurisdiccional de la ley perdió vigencia al constituirse Tierra del Fuego
en provincia, y quedó circunscrito al ámbito de la Ciudad de Buenos Aires.
14 Esa primera comisión estaba conformada así: Presidente: Santos Colabella;
Vicepresidente: Agustín Cafferata; Secretario General: Osvaldo Denker; Secretaria
de Asuntos Profesionales: Edelma Enriquez; Tesorero: Juan Carlos Peña; Vocales
titulares: Edgardo Margiotta, Bernardo Maresca, Luis Stuhlman, Jorge Taiana, Jorge
Ocaña y Mónica Cambiasso; Vocales suplentes: Carlos Dasso, Alicia Toribio, María
Elina Estebanez, Hugo Balmaceda, Esteban Castro y Liliana Findling. El primer
Tribunal de Ética y Disciplina estuvo integrado por: Miembros titulares: Atilio 
multáneamente durante cuatro años y medio, y el Colegio debió prestar primero y alquilar después, su sede al Consejo para que pudiera funcionar. En esos años, el Colegio empezó a registrar problemas para cobrar a lxs socixs, pero no descendió su nivel de actividad. El Consejo iniciaba sus actividades y comenzó a matricular al año siguiente.

Algunxs de quienes se vincularon al CPS lo hicieron por previa relación con el Colegio. La decisión del cierre del CGS en 1994 fue, según Alicia Palermo, discutida y resistida porque muchxs no querían que deje de funcionar; "pero la gente no pagaba las dos cosas"(Entrevista, julio de 2019).

La búsqueda de organizaciones, como el CPS y el CSPBA, para reunir a todxs lxs sociólogxs como profesionales, de regular el ejercicio de la profesión y sus condiciones, de ser las "organizaciones de base" que mantengan vínculos con organismos públicos y privados, que ya podían evidenciarse en los fundamentos para la creación de la FNS, se mantuvo durante los años posteriores y hasta la actualidad. Los colegios fueron encontrando muchas dificultades para lograrlo e incluso han lidiado desde sus comienzos ${ }^{15} \mathrm{y}$ hasta hoy con la dificultad para hacer que lxs sociólogxs se asocien y/o matriculen, incluso cuando las leyes de distintas jurisdicciones establecen la obligatoriedad de la matriculación.

Esta dificultad de matriculación repercute en la escasa llegada de las organizaciones a un espectro amplio de sociólogxs trabajando (algunxs ni siquiera saben que existen), pero también

Borón, Catalina Wainerman y Daniel Filmus; Miembros suplentes: Hugo Mercer, Ernesto Villanueva y Raúl Bisio.

15 En las Actas de Comisión Directiva del CPS del año 1991 puede constatarse que buena parte de las reuniones se las llevaba el diseño de estrategias de difusión e información a sociólogxs e instituciones respecto de la "importancia y legalidad de la matriculación", porque esta no adquiría el ritmo de crecimiento que esa CD esperaba, lo cual traía, en primera instancia, problemas financieros. 
en recursos materiales y financieros escasos, porque se sostienen con el dinero de las matrículas.

La creación de nuevas asociaciones profesionales a partir de la década del 80 contrasta, entonces, con las dificultades que fueron encontrando en años posteriores para lograr representatividad entre sociólogxs. A medida que las diversas asociaciones de graduados lograban transformarse en colegios o consejos creados por leyes que regulan el ejercicio profesional en sus jurisdicciones, encontraban cada vez más difícil de lograr el objetivo de constituirse en representativas de los intereses del conjunto de sociólogxs. También encontraban serias dificultades para sostener la existencia de las asociaciones. Si bien en los primero años 90 comenzó a funcionar el CPS, lo hizo con una matriculación menor a la que sus miembros esperaban y con los consecuentes problemas financieros. En los mismos años, se decidió la disolución del CGS.

La década siguiente registró, sin embargo, la creación de nuevas organizaciones. En 2000 se creó la Asociación de Sociólogos de la República Argentina (ASRA); en 2005, el Colegio de Sociólogos de Santiago del Estero (por ley); en 2009, la Asociación de Profesionales de Sociología de Mendoza y la Asociación Argentina de Sociología (AAS); en 2010 la Federación Argentina de Sociología (FAS) ${ }^{16}$. De ellas, la única de la que no existen indicios de actividad en la actualidad es la última mencionada.

El ritmo de creación de asociaciones locales, regionales y nacionales en los últimos casi 20 años puede resultar llamativo, a juzgar por las dificultades para atraer la participación. Algunas buscan nuclear a los mismos grupos o segmentos de sociólogxs, como las organizaciones de carácter nacional. Si bien quienes conducen ASRA y AAS diferencian los objetivos de ambas, iden-

\footnotetext{
16 Fuentes: registros de campo en presentaciones de miembros del CPS y CSPBA (agosto, 2017; mayo, 2019) y páginas web: http: / / sociologosmendoza.blogspot.com; www.aasociologia.org.ar; www.colesociologossj.blogspot.com
} 
tificándose como asociación "profesional" la primera ${ }^{17}$,y como organización "de carácter académico" la segunda, la existencia simultánea de organizaciones que se ocupan de aspectos que hacen a las tareas y saberes de un mismo grupo profesional y que pretenden representar los intereses de lxs sociólogxs de todo el país abre al menos una pregunta que guiará próximas indagaciones: ¿qué significados adquiere, desde las perspectivas de quienes las impulsan y también de quienes eligen no involucrarse, el funcionamiento simultáneo y paralelo de asociaciones en un escenario en el que lxs sociólogxs del país no han mostrado, en las últimas décadas, mucho interés por asociarse y pagar cuotas y matrículas?

Vale decir que las dificultades para sumar matriculados no impidieron que los colegios y consejos continuaran realizando actividades dirigidas a la "comunidad de sociólogxs";sobre todo a partir de la década del 2000, donde las iniciativas aumentaron, y una muestra de ello es la creación en 2003 de la Revista Argentina de Sociología, por parte del CPS. Esta revista logró instalarse como órgano de publicación con prestigio y se editó semestralmente hasta 2009; luego, tuvo publicaciones discontinuas y fue relanzada en 2016. También en el CPS se creó el Instituto de Investigaciones Sociológicas (IDIS), centro asociado de CLACSO. Por su parte, el CSPBA organizó en esa década dos Congresos de Sociólogos de la Provincia de Buenos Aires: uno en 2009, realizado en La Plata, y otro en 2011 en Mar del Plata.

Con todo, la promoción de legislación y la creación de nuevas asociaciones de diversa índole - algunas funcionan como colegios que matriculan y controlan, otras como organizaciones civiles que nuclean voluntariamente- han sido las estrategias

\footnotetext{
17 Quienes integran ASRA y otras asociaciones como CSPBA y CPS tienen trayectorias laborales y se identifican con expertise (según el término que algunxs de ellxs usaron en distintas ocasiones) diversas. Gestión de políticas públicas, consultoría de mercado y opinión, encuestas y estadísticas, capacitación, trabajo académico en su mayoría docente.
} 
privilegiadas por quienes integran las asociaciones para constituirse en actores activos en la definición de en qué consiste "la sociología como profesión" y como "ocupación".

\section{"Pluralidad", organización gremial y tensión entre "profesión" y "academia"}

El funcionamiento colegiado y la legislación continúan siendo apuestas de las asociaciones para "jerarquizar la profesión"; pero para comprender las maneras en que entienden esa "jerarquización" y buscan lograrla, es fundamental considerar el cambio que se fue produciendo en las maneras de nombrar y construir por parte de quienes integran los colegios, las relaciones y diferencias entre "el ejercicio profesional"—identificado cada vez más con el trabajo fuera de las universidades- y "la academia" - identificada con la dedicación exclusiva en universidades y centros de investigación.

En agosto de 2017 presencié la actividad de una mesa de las XII Jornadas de Sociología de la UBA, titulada "Formación e inserción laboral de graduados de sociología". Allí Alejandro Terriles, entonces ejerciendo un cargo en el CPS, tuvo intervenciones que llamaron mi atención, porque mostraban enojo y marcaban diferencias y un terreno propio: hablaba del "ejercicio de la profesión", los marcos legales y las distancias con "la academia", diciendo que hay "bajo reconocimiento de la comunidad universitaria de las entidades profesionales, asociado a la falta de información y a que la currícula no incorpora temas del ejercicio profesional" (Registro de campo, 24/8/2017). Después de ello, en uno de nuestros primeros intercambios, Terriles me dijo: "Lo nuestro es político y expresamos un complejo mosaico de miradas sobre la tensión entre la profesión y la academia" (Mensaje de whatsapp, 10/4/2018).

¿Qué sentidos construyen estxs sociólogxs sobre la política y su relación con la actividad de las asociaciones? ¿En qué consiste la "tensión entre profesión y academia" desde la perspectiva 
de quienes participan en las asociaciones? En una entrevista personal, Terriles avanzó en describir esta tensión:

Somos los únicos que en principio nos dedicamos a pensar y accionar, no solamente pensar y discurrir, sobre la actividad profesional, porque la mayoría del grupo de conducción es básicamente gente que viene con una formación (...) nuestros derroteros laborales son la sociología práctica, no tanto la reflexión académica.Son asesores políticos, gente que viene con una experiencia en sindicatos, en organizaciones sociales, en ONG, bueno, tenemos toda una cosmovisión que un poco es la antítesis de la formación académica universitaria. (...) Las carreras de sociología tienen una visión muy centrada, muy endogámica, muy como de toda la reflexión intelectual es adentro de las universidades, facultades e institutos de investigación. (Entrevista, abril de 2018)

Analizar las formas en que Terriles nombra la "tensión" es relevante, porque son significativas para comprender los posicionamientos de quienes definen las diferencias entre "academia" y "profesión" desde los lugares y actividades que obtienen "poco reconocimiento". De un lado, en las asociaciones se dedican a "pensar y actuar", al contrario de quienes se dedican a "pensar y discurrir" en la academia. Las universidades y centros de investigación son caracterizadas por su endogamia, porque "los académicos" conciben que la reflexión intelectual solo ocurre allí. A ello, Terriles opone la "acción" y la "práctica" como valores del trabajo de sociólogxs con recorridos no centrados en la investigación y los espacios académicos.

Desde estas asociaciones, se busca valorizar el trabajo y el saber de las personas con esos derroteros. Esa valoración se presenta, en palabras de Terriles, como una forma de "dar cobertura a todos los profesionales que trabajan en sociología en la república argentina" (Registro de campo, presentación de Panel a cargo de 
asociaciones profesionales de sociología en la Feria Internacional del Libro de Buenos Aires, 8/5/2019).

Otra manera de dar valor al trabajo es romper con la "endogamia" de las universidades."[En el grupo que conduce CPS, CSPBA y ASRA ${ }^{18}$ ] hay pluralismo de ideas, desde el punto de vista de lo político y lo ideológico, pero unidad de criterios en cuanto a cómo pensamos la actividad profesional" (Entrevista a Alejandro Terriles abril de 2018).

La tensión entre "academia" y "profesión" parece residir, para estxs sociólogxs, en las acciones que en cada espacio tienen lugar en relación a la "jerarquización de la profesión": en "la academia" encuentran sectarismo, endogamia y desvalorización "del ejercicio práctico"; en las asociaciones, las personas con las que dialogué en las visitas realizadas, definen su política de conducción como de "pluralidad política e ideológica" y "unidad" para "dar cobertura a los colegas" (Registros de campo de abril, junio y julio de 2018).

A la hora de pensar la actividad profesional, el objetivo sigue siendo, como desde los años 80, la organización gremial. Durante la misma conversación, Terriles agregó que "la finalidad social de las instituciones es justamente generar un campo regulado donde toda esa gente pueda tener un mercado de trabajo medianamente asegurado" (Entrevista, abril de 2018).

Regular "el campo" y "asegurar" un mercado parecen ser las maneras en que, desde estas posiciones, buscan responder al problema de la "ocupación", de hacer de "la profesión" lo que les provee sustento, sobre todo a quienes se desempeñan en espacios "poco valorados" por "los sociólogos académicos.

\footnotetext{
18 Con algunas variantes, el mismo grupo, llamado Colectivo Sociológico Plural, ganó las elecciones en el CPS y el CSPBA desde 2014 y va por el tercer periodo de conducción de ambas entidades. Algunxs integran también la agrupación GraduadxsXGraduadxs, definido por ellxs como "nuestro espacio de militancia universitaria", que compite en elecciones del claustro de graduados en la Facultad de Ciencias Sociales de la UBA, de la cual depende la Carrera de Sociología.
} 
Pero hay algo más. La pluralidad que reclaman y de la que a veces se jactan lxs sociólogxs de las asociaciones no es solo política e ideológica, o teórica e intelectual. En el persistente debate sobre qué es dedicarse a la sociología, estxs sociólogxs se presentan sin adscripciones genealógicas, pero reclamando reconocimiento como productores de conocimiento sociológico. En una conferencia dictada en la Feria Internacional del Libro de Buenos Aires y titulada "Aportes de lxs profesionales en sociología a la resolución de los problemas de la Argentina contemporánea", Daniel Natapof, sociólogo rionegrino y actual presidente de ASRA, se refirió a la "producción sociológica" de los "profesionales":

Estamos los expertos, técnicos, ingenieros sociales que hacemos encuestas, focus groups, escribimos discursos, hacemos diseño y planeamiento de políticas públicas de diversa índole, elaboramos diagnósticos, asesoramos, hacemos consultorías, hacemos evaluación de programas sociales. No somos científicos, ¿sí? Somos los que producimos conocimiento socialmente útil. Tenemos tiempos acotadísimos, pocos recursos humanos y materiales, tenemos que viajar, a veces arreglarnos con dos pesos. Hacemos trabajos gratuitos por cuestiones estratégicas, a veces por convicción. Tomamos riesgo a la hora de dar una opinión, que no tiene el tratamiento académico que quisiéramos, pero hay que hacerlo. No tenemos tiempo para buscar un marco teórico a veces, y al decisor político además no le interesa, y muchas veces permanecen ocultas porque la mayor parte del trabajo que hacemos es confidencial. Porque no tiene validación científica y porque el mundo académico mayormente lo ignora, porque es trabajo no científico. Pero es producción sociológica plena, abundante y muy rica, ¿sí? Siempre he pensado que es triste que haya una tradición academicista que se prive de integrar conocimientos, experiencias e inhiba una relación dialéctica entre teoría y praxis.(Registro de campo, mayo de 2019) 
Al parecer, muchxs de lxs sociólogx buscan, al participar de estas asociaciones, el reconocimiento y la valoración del trabajo que la "tradición academicista" les estaría negando. En estas afirmaciones, realizadas públicamente o en conversaciones personales, se manifiesta un malestar con algo que lxs sociólogxs no tienen, la sociología tampoco, y el profuso desarrollo en universidades y centros de investigación científica no hacen y no ofrecen a "los profesionales". La sociología, dicen, requiere ser llevada a que "la conozcan" y que políticos y tomadores de decisiones en general —en el Estado, empresas y organizaciones civiles-“la usen".

Quienes forman parte de colegios y asociaciones profesionales están disputando sentidos de la "profesión" y formas de valoración del trabajo. Lo hacen desde instituciones en las que "hacen política" para "promover una sociología amigable, una sociología para la gente" (expresión de la actual presidenta del CPS, Mercedes Jones, en el panel realizado en la Feria Internacional del Libro de Buenos Aires. Registro de campo, mayo de 2019) y ofrecer beneficios "a los colegas". Recibiendo a todo el que esté interesado en "aportar algo", sin importar su adscripción política, teórica o intelectual.

\section{Conclusiones}

Los análisis presentados a lo largo de este escrito permiten entender que, en el terreno de la pregunta por qué es dedicarse a la sociología, quienes participan en las asociaciones, colegios y federaciones, no buscan dar sentido y contenido a ese interrogante recurriendo a tradiciones teóricas ni a genealogías, sino a otros elementos que me propuse describir y comenzar a explorar en este artículo. La organización gremial, la valoración de la "sociología práctica", la legislación, la acción política en las asociaciones y la búsqueda de regulación estatal serían algunas de las claves que permiten comprender los posicionamientos construidos por quienes buscaron en la asociación una forma de "jerarquizar la profesión". 
Para entender estos posicionamientos, fue preciso reconstruir las maneras en que estas instituciones, y quienes participaron de ellas, fueron dando sentido en diversos momentos y contextos a "la profesión" y al rol que en su desarrollo fueron buscando tener los colegios, consejos, federaciones y asociaciones. En este sentido, si comprendemos a la profesión como una de las formas históricamente posibles de organización de una ocupación y, particularmente, la creación y sostenimiento de asociaciones profesionales como una estrategia para consolidar esa forma de regulación (Evetts, 2010; Friedson, 2001; González Leandri, 1999; Johnson, 1995), tenemos que tomar en cuenta que, en el campo sociológico argentino, la idea misma de "profesión" está en disputa. En esta disputa, solo algunos grupos de sociólogxs buscaron la legitimación de su ocupación a través de la asociación profesional. Esos grupos no han sido dominantes en el campo, aunque en algunos momentos estas instituciones hayan sido espacios convocantes y significativos. Si recuperamos la experiencia de los años 80 desde la perspectiva de quienes participaban de Colegios o Asociaciones profesionales, nos encontramos con relatos que hablan de una fuerte actividad, de una "revitalización" y de una búsqueda de "recomponer institucionalmente" a la sociología a partir de la organización gremial, incluso anterior a las iniciativas surgidas desde las universidades.

Sin embargo, es interesante encontrar que en las investigaciones publicadas sobre la profesionalización e historia de la sociología en Argentina, la década de1980 aparece caracterizada por dos procesos principales que habrían marcado el desarrollo del campo: el proceso de diversificación del mercado laboral y el de "refundación" de la carrera en la UBA (Blois, 2012, 2018; Sidicaro, 1993). Pero las asociaciones profesionales son escasamente incorporadas a los análisis de este periodo y de los procesos mencionados, aunque se trata de actores siempre existentes $\mathrm{y}$ activos.

Asimismo, vimos que la dificultad para lograr que lxs sociólogxs se matriculen en los colegios y consejos que regulan el 
ejercicio persistió desde los primeros tiempos. Al respecto, José Casco (2018) identificó esta dificultad con "una particularidad argentina": "No es en la asociación profesional donde los sociólogos buscan refugiarse para la salvaguarda de su profesión ni tampoco un espacio constructor de su identidad [y] estas asociaciones poco pudieron hacer para proteger el ejercicio de la profesión" (p. 30).El objetivo de este artículo es aportar descripciones que permitan dar mejor cuenta de la heterogeneidad del campo sociológico argentino. Esta heterogeneidad no se define únicamente por las tradiciones teóricas y los linajes académicos. La realidad es que grupos de sociólogxs eligieron la vía de las instituciones profesionales, la colegiación y control de la matrícula por ley, y lo hicieron en distintas oportunidades, a juzgar por las varias jurisdicciones en las que promovieron y lograron que una ley se sancione. Por ello, considero que resulta fundamental conocer las perspectivas de quienes han constituido estos grupos, así como sus actividades dentro de las organizaciones para comprender cuál es su participación en las disputas por la definición de qué es dedicarse a la sociología y cuáles son los sentidos de "la sociología como profesión".

Las asociaciones aún pelean por una ley nacional, por la producción de datos estadísticos, por la revisión de las incumbencias profesionales, por la exigencia de parte de los empleadores uno de los principales, el Estado- de matriculación y por políticas de regulación. ¿Pelean, en definitiva, por la injerencia estatal en el control del ejercicio? ¿La colegiación es, en el caso de lxs sociólogxs argentinxs, la búsqueda de la autoprotección o de la protección por parte del Estado? ¿Qué ha sido y qué es el Estado para lxs sociólogxs argentinxs a la hora de buscar o constituir espacios para construir su identidad?

\section{Referencias}

Beltrán, G. (2010).Las ciencias sociales y el surgimiento de un mercado del saber experto. Las bifurcaciones de la sociología ar- 
gentina en el final del siglo XX. En Rubinich \&Beltrán (Eds.) ¿Qué hacen los sociólogos?(pp. 49-72). Buenos Aires: Aurelia Rivera.

Bialakowsky, A., Martín de, H., Macri, M. R., Filmus, D.\& Caracciolo, A. (1982).Espectro ocupacional del licenciado en sociología en el medio profesional argentino(Primer Informe). Buenos Aires: Carrera de Sociología, Universidad de Buenos Aires, Colegio de Graduados en Sociología.

Blanco, A. (2006).Razón y modernidad. Gino Germani y la sociología en la Argentina. Buenos Aires: Siglo XXI.

Blois, P. (2012).Obligados a elegir "entre el sacerdocio y la prostitución". Socialización universitaria y prácticas profesionales de los sociólogos de la UBA.(Tesis Doctoral inédita). Universidad de Buenos Aires, Buenos Aires.

Blois, P. (2018). Medio siglo de sociología en Argentina. Ciencia, profesión y politica (1957-2007). Buenos Aires: Eudeba.

Blois, P. (2019). Sociología y regímenes autoritarios. La carrera de sociología de la UBA y los circuitos académicos alternativos durante "los años de plomo". Sociohistórica, (43), 1-19. Disponible en: https://doi.org/10.24215/18521606e071

Caracciolo, A.(2008). Itinerarios de la sociología en la década del ochenta: La actuación del Colegio de Graduados y Especialistas en Sociología de Córdoba". Trabajo presentado en V Jornadas de Sociología de la UNLP, FAHCE-UNLP, La Plata, Argentina

Carrera, C. (2018).Enseñanza, cuestionamientos y herencias en la formación de sociólogxs profesionales en los años '50 y '60. Trabajo presentado en las VIII Jornadas de Etnografía y Procesos Educativos, IDES, Buenos Aires.

Casco, C. (2018).Una guerra de movimientoso la historia de la sociología en clave generacional. Apuntes de investigación del CECYP, (30), 129-132. Disponible en: http:/ / www.apuntescecyp.com.ar/index.php/apuntes/article/view/685

Díaz, D. (2013).La trayectoria del sociólogo Alfredo Poviña luego de la derrota frente a Gino Germani (1955-1983). Transformaciones politicas-culturales en la Argentina pos peronista y el desarrollo del campo sociológico en Córdoba. Trabajo presentado en $X$ Jornadas de Sociología, Universidad de Buenos Aires, Buenos Aires. 
Evetts, J. (2012).Professionalism: value and ideology. Sociopedia.isa, 1-10. Disponible en doi: $10.1177 / 205684601231$

Freidson, E. (2001). La teoría de las profesiones. Estado del arte. Perfiles Educativos, XXIII(93), 28-43.

González Leandri, R. (1999). Las profesiones. Entre la vocación y el interés corporativo. Fundamentos para su estudio histórico. Madrid: Catriel.

González Leandri, R. (2006). Campos e imaginarios profesionales en América Latina. Renovación y estudios de caso. Anuario IEHS,(21), 333-344.

Grupo Taller Pensar la Facultad. (2009). Aprendiendo sociología. La impronta de la Carrera en la experiencia de los estudiantes. Buenos Aires: Facultad de Ciencias Sociales, Universidad de Buenos Aires.

Izaguirre, I. (2010). El perfil de un maestro. Gino Germani, fundador de la sociología en Argentina, 30 años después. En Mera, C. \& J. Rebón (Coords.),Gino Germani, la sociedad en cuestión:antología comentada. Buenos Aires: Consejo Latinoamericano de Ciencias Sociales.

Johnson, T. (1995). Governmentality and the institutionalization of expertise. En T. Johnson, G. Larkin \& M. Sacks (Eds.),Health professions and the state in Europe(pp. 4-13). London: Taylor\& Francis.

Jorrat, R.\&Sautu, R. (Comps.). (1992). Después de Germani. Exploraciones sobre estructura social de la Argentina.Buenos Aires: Paidós

Maderna, F. (2000). Entrevista a Alcira Argumedo. En H. González (Comp.) Historia crítica de la sociología argentina. Los raros, los clásicos, los científicos, los discrepantes.(pp 479-492). Buenos Aires, Colihue.

Mera, C. \& Rebón, J. (2010). Actualidad y retrospectiva del pensamiento de Gino Germani. En C. Mera \& J. Rebón (Coords.). (2010). Gino Germani, la sociedad en cuestión: antología comentada. Buenos Aires: Consejo Latinoamericano de Ciencias Sociales

Pennycook, A. (2010). Language as a local practice. Londres: Routledge

Pereyra, D. (2005). International Networks and the Institutionalization of Sociology in Argentina (1940-1963). (Tesis de Doctorado). School of Social Sciences and Cultural Studies, University of Sussex, Brighton. 
Rubinich, L.(2010). Cómo relatar aquello que hacen los sociólogos. En L. Rubinich \& G. Beltrán (Eds.) ¿Qué hacen los sociólogos? (pp. 9-48). Buenos Aires: Aurelia Rivera.

Rubinich, L. \& Beltrán, G.(Eds.). (2010). ¿Qué hacen los sociólogos? Buenos Aires: Aurelia Rivera.

Sarfatti Larson, M. (1980). Proletarianization and educated labor. Theory and Society, 9(1), 131-175.

Sarfatti Larson, M. (1988). El poder de los expertos. Ciencia y educación de masas como fundamento de una ideología. Revista de Educación,(285), 151-189.

Sidicaro, R.(1993). Reflexiones sobre la accidentada trayectoria de la sociología en la Argentina. Cuadernos Hispanoamericanos, (517519), 65-76.

Terriles, A. (2015). Reseña Institucional. Disponible en: www.colsociologospba.org.ar/colsociolospba2015/historia.htm

Tortti, C.\&Chama, M. (2003). Constitución y desarrollo de la carrera de sociología de la UNLP. Entrevista a Alfredo Pucciarelli. Revista Cuestiones de Sociología, (1), 135-173.

Verón, E. (1974). Imperialismo, lucha de clases y conocimiento: 25 años de sociología en la Argentina. Buenos Aires: Tiempo Contemporáneo.

Vommaro, G.(2008). Lo que quiere la gente.Buenos Aires: Prometeo.

Wagner, P., Weiss, C.,Witrock, B. \&Wollman, H. (Comps.).(1999). Ciencias sociales y estados modernos. Experiencias nacionales e incidencias teóricas. México D. F.: Fondo de Cultura Económica.

\section{Fuentes}

Actas de la Federación Nacional de Sociólogos (FNS) de 1983 a 1985.

Actas del Colegio de Graduados en Sociología (CGS) de 1991 a 1994.

Actas de la Comisión Directiva del Consejo de Profesionales de Sociología (CPS), de 1990 a 1992.

Ley 23553 "Ejercicio Profesional de la Sociología". Disponible en http: / / servicios.infoleg.gob.ar /infolegInternet/anexos/20000-24999/20990/norma.htm 


\section{Entrevistas:}

Palermo, Alicia, 4 de julio de 2019.

Terriles Alejandro, 13 de abril de 2018.

Wainerman Catalina, 5 de septiembre de 2018. 